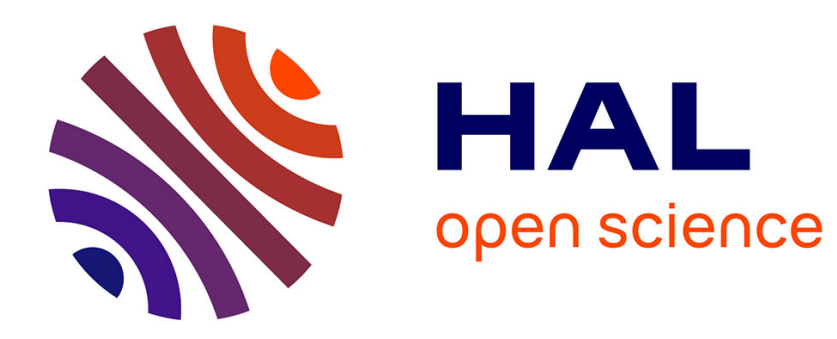

\title{
Directional Order and Diffusion Aftereffect
}

Louis Néel

\section{To cite this version:}

Louis Néel. Directional Order and Diffusion Aftereffect. Journal of Applied Physics, 1959, 30 (4), pp.S3-S8. 10.1063/1.2185960 . hal-02887449

\section{HAL Id: hal-02887449 \\ https://hal.science/hal-02887449}

Submitted on 2 Jul 2020

HAL is a multi-disciplinary open access archive for the deposit and dissemination of scientific research documents, whether they are published or not. The documents may come from teaching and research institutions in France or abroad, or from public or private research centers.
L'archive ouverte pluridisciplinaire HAL, est destinée au dépôt et à la diffusion de documents scientifiques de niveau recherche, publiés ou non, émanant des établissements d'enseignement et de recherche français ou étrangers, des laboratoires publics ou privés. 


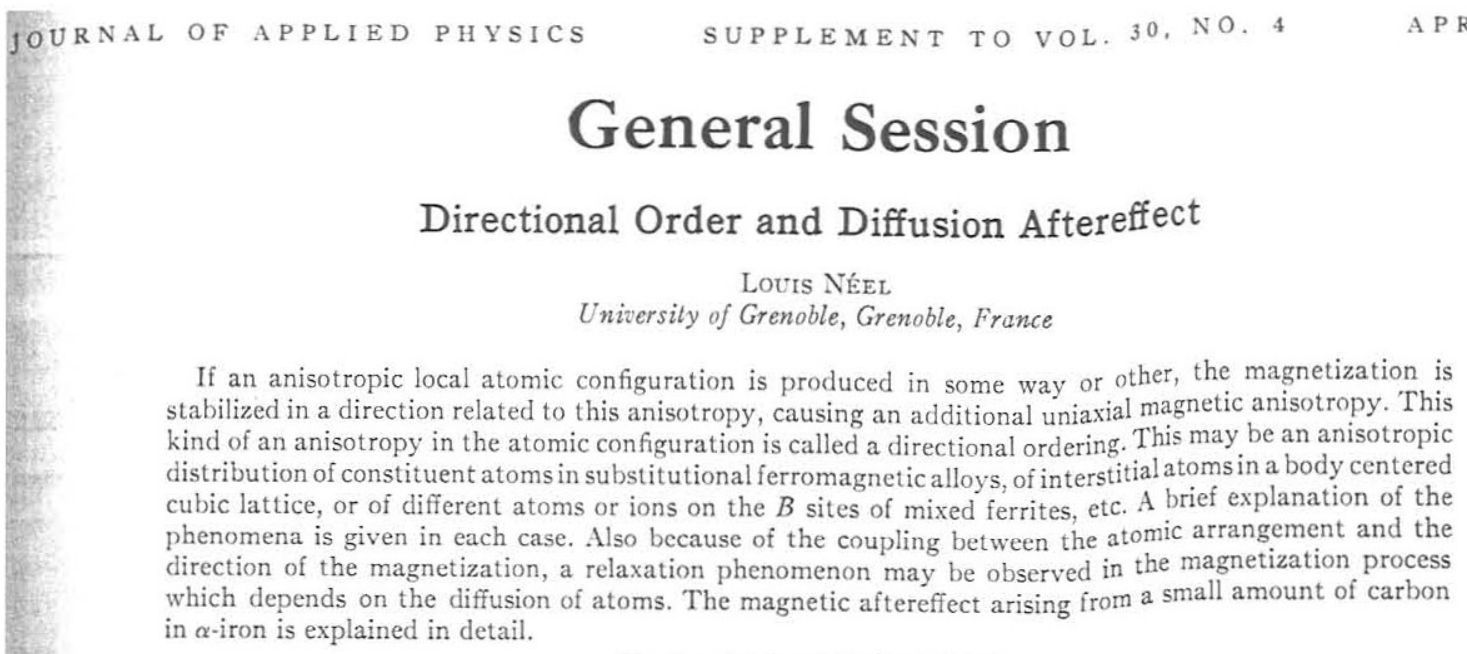

\section{LOCAL CONFIGURATIONS OF NEIGHBORING ATOMS}

$\mathrm{T}^{\mathrm{H}}$ HE free energy of a ferromagnetic substance depends to a great extent on the local configurafion, i.e., on the nature and position of the nearest neighbors of a given atom. It is well "known that the interactions between moment carrying atoms are of two kinds; namely, very intense and isotropic interactions due to exchange or superexchange, and considerably weaker and anisotropic interactions such as, for example, those due to magnetic dipole coupling.

Interactions of the first kind, overcoming the thermal fluctuations, establish a short-range order in the orientation of atomic moments, responsible for the spontaneous magnetization. The stronger these interactions, the higher the Curie temperature at which the spontaneous magnetization vanishes. Changes in the local configuration alter the free energy and the Curie temperature. But from a practical standpoint the effects of these changes on the technical magnetization may be neglected, since variations in the technical magnetization are due, in the last analysis, to a rotation of the magnetization vector, and since this rotation does not alter the energy, in view of the isotropic character of the interactions considered.

Thus the technical magnetization laws are almost exclusively dependent on the relatively weak and anisotropic interactions, which determine the magnetocrystalline energy and on the orientation of the axes of easy magnetization. Before proceeding further, let us review a few examples of the changes that may occur in the local configuration.

\section{ANISOTROPY OF LOCAL CONFIGURATIONS}

(a) Let us consider as a first example a solid solution of cubic structure whose two constituents $A$ and $B$ (one of which at least is magnetic) have respective atomic concentrations $C_{A}, C_{B}\left(C_{A}+C_{B}=1\right)$. The local atomic concentration of neighboring atoms about a given occupied site, say an $A$ atom, is in general anisotropic, but the mean local configuration (obtained by averaging over all $A$-occupied sites) is in general isotropic. Defining the line segment joining two neighboring atoms as a link, there will be 3 types of link, referred to as $A-A, A-B$, and $B-B$ links, respectively. The probabilities for an arbitrary link to belong to one of these 3 classes are $C_{A}{ }^{2}, 2 C_{A} C_{B}$, and $C_{B}{ }^{2}$, respectively. It is easy to see that after some suitable "anisotropic treatment," of a kind to be further specified, these respective probabilities may be altered, and made dependent on the orientation of each link, relative to some axis $D$ associated with that particular treatment. One thus establishes what may be called a directional ordering due in the last analysis to the anisotropy of the mean local configuration. This is a short-range order in general. However when this directional order is very strong, it becomes a genuine long-range order, which we shall call an orientation superlattice. This orientation superlattice is characterized by a true cell, each site of which is occupied by a well-defined atom. It is uniaxial and may be rhombohedral or tetragonal.

Let us note in particular that vacancies in the crystal lattice may play the same role as the foreign atoms do.

(b) A second example is that of interstitial atoms in a ferromagnetic crystal lattice. This can be exemplified by carbon (or nitrogen, or boron) atoms in the b.c.c. structure of $\alpha$-iron. Let us assume, for example, that these interstitial atoms are located midway between two neighboring iron atoms, i.e., at the midpoint of a link. There are three classes of links, i.e., three classes of sites for the carbon atoms, corresponding to the three mutually perpendicular axes of the lattice, and these classes are in general equally populated. Appropriate anisotropic treatments may lead to unequal populations and consequently to a macroscopic anisotropy. In this and the preceding cases, we are dealing with an anisotropy due to the mean local configuration.

(c) A third example is that of compounds with cubic structure where two groups $A$ and $B$ of different atoms can occupy sites whose immediate neighborhood exhibits a symmetry inferior to the total symmetry (cubic) of the lattice. This is the case, for example, for a mixed spinel ferrite in which two kinds of atoms are distributed over the $B$ sites. Each $B$ site is surrounded 
with six oxygen atoms symmetrically placed, three by three with respect to a ternary axis. There are thus three different classes of $B$ sites, which are in general equally propulated in atoms $A$ and $B$. The mean local configuration is then isotropic, but is no longer so after an anisotropic treatment.

There are other examples, but the three cases mentioned are typical: Néel was first to describe the first two, while the third was described by Bickford.

\section{TREATMENTS LEADING TO ANISOTROPY}

We have just reviewed a number of examples showing the possibility of creating macroscopic anisotropy through the superposition of local anisotropic configurations. To establish this anisotropy it is necessary to submit the sample to a treatment such that the various local configurations correspond to different energies. What kind of treatments are these? A first procedure, of the mechanical type, consists in straining the crystal in anisotropic fashion by means of appropriate mechanical constraints (tension, compression, etc., …). A second procedure, of the magnetic type, consists simply in magnetizing to saturation a ferromagnetic sample, i.e., aligning the spontaneous magnetizations along the same direction, thus creating a magnetic anisotropy.

\section{MECHANISM OF THIS PHENOMENON : RELAXATION}

The mean anisotropy of local configuration, produced by an anisotropic treatment, results in fact from actual displacements of the atoms (sometimes of electrons) constituting the sample. In cases (a) and (b), atoms $A$ and $B$ exchange sites either directly or through vacancy displacement. In case (b) interstitial atoms jump from one position to another. In both cases, the atoms must overcome a potential barrier and this implies an activation energy $W_{a}$. Equilibrium in the local configuration will be reached with a time constant $\theta$ given by a formula of the type

$$
\theta=C \exp \left(W_{a}\right) / R T
$$

where factor $C$ varies slowly with $T$ and $W_{a}$. For true equilibrium to be obtained, it is necessary that the order of magnitude of $\theta$ be the same as that of the duration of the experiment: the temperature must therefore reach a value equal to or larger than some temperature $T_{a}$.

Experiments show, however, that a unique time constant is a rather exceptional case. What is generally observed is a spectrum of time constants lying within an interval of variable magnitude. Thus in an Fe sample with an atomic carbon concentration of the order of $10^{-5}$, Brissonneau observed a spectrum of time constants within an interval $\left(\theta_{1}, \theta_{2}\right)$ such that $\theta_{2} / \theta_{1}=25$. The temperature $T_{a}$, for which the mean time constants are comparable to the duration of the experiments, say $1 \mathrm{~min}$ for example, is extremely variable. In solid solutions $A-B$ of type (a), this temperature reaches values ranging from 700 to $900^{\circ} \mathrm{K}$. For interstitial atoms, such as carbon in $\alpha$-iron, its value is $250^{\circ} \mathrm{K}$. For exchange electrons, such as is the case between $\mathrm{Fe}^{++}$and $\mathrm{Fe}^{+++}$in magnetite, its value is $100^{\circ} \mathrm{K}$.

\section{ORIENTATION SUPERLATTICES}

The above discussion suggests that the implications of the phenomena considered may be of two kinds.

By means of a mechanical or a magnetic treatment applied at a temperature higher than $T_{a}$ (at which) temperature the atoms have a greater mobility), followed by a sudden quenching to a temperature $T_{i}<T_{a}$ it is possible to maintain the atoms in their initial positions and thus "freeze" the anisotropy of local configurations. This anisotropy, although generally very weak, may be detected and accurately measured by magnetic methods. One may for example place a diskshaped sample in an intense and uniform magnetic field parallel to its plane, and measure the torque exerted on the disk as a function of the azimuthal angle. The anisotropy is thus due to the formation of axes of easy magnetization resulting from a directional ordering due a mechanical, or a magnetic heat treatment. From an experimental standpoint, the formation of easy axes of magnetization by a magnetic heat treatment has been known for a long time, but the interpretation of these phenomena as well as the theory of directional ordering were given for the first time in 1953 by Néel. ${ }^{1}$ A similar theory was later formulated by Taniguchi. ${ }^{2}$ As for the mechanical heat treatments, their effects described in 1954 were observed only recently. ${ }^{3}$

\section{MAGNETIC AFTEREFFECT DUE TO DIFFUSION}

Instead of considering the local configurations frozen by quenching, one may perform the experiments at temperatures nearly equal to or higher than $T_{a}$. In this case an external field, altering the direction of spontaneous magnetization, alters also the local equilibrizm configurations. However, due to the changes in the direction of the magnetization with time, equilibrium cannot be obtained, and thus appears a timelag involving an energy loss. This is one of the causes of magnetic aftereffect (sometimes called magnetic diffusion). The magnetic aftereffect is here related to the displacement of atoms or electrons inside a sample, i.e., to diffusion processes. From this comes the name diffusion aftereffech, that we have given to this phenomenon, in agreement with an earlier paper ${ }^{4}$ where we had for the first time pointed out the existence of two distinct magnetic aftereffects, namely, the diffusion aftereffect just described and the aftereffect due to thermal fluctuations. The latter is due, in particular, to irregular thermal variations of

${ }^{1}$ Louis Néel, Compt. rend. 237, 1468 and 1613 (1953).

2 S. Taniguchi and M. Yamamoto, Sci. Repts. Research Insts. Tohoku Univ. Ser. A, 6, 330 (1954); Ser. A, 7, 269 (1955).

${ }^{3}$ Louis Néel, Compt. rend. 238, 305 (1954); R. Vergne, Comn Colloque Int. Magnétisme Grenoble, July 2-6, 1958.

'Louis Néel, J. phys. radium 12, 339 (1951). 
the internal dispersion field, analogous to a Brownian motion.

A close relationship thus exists between diffusion aftereffect and orientation phenomena, i.e., the formation of easy axes by heat treatment in a magnetic field. Both are but different aspects of the same phenomenon; namely, the mean anisotropy of local configuration (directional ordering is only a special case).

\section{AN EXPRESSION FOR THE ENERGY}

To develop the theory, it is necessary to make some simple assumptions on the energies involved. In case (a) for example we assumed that the total energy of the system due to the local configurations is simply the sum of the energies of the various links. One thus associates with each elementary link an energy $w$ given by

$$
w=g_{1}(r) P_{2}(\cos \varphi)+g_{2}(r) P_{4}(\cos \varphi)+\cdots,
$$

where the $P_{n}$ are Lengendre polynomials, $\varphi$ the angle between the link and the local direction of spontaneous magnetization, and $g_{1}(r), g_{2}(r), \cdots$ are functions of the distance $r$ between the two atoms of the link, $r$ depending on the nature of the link $(A-A, A-B$, or $B-B)$. A simpler form of $w$ could be $w=(l+m \delta r) \cos ^{2} \varphi$, where $\delta r$ represents possible variations of $r$. Such a formalism has the advantage of relating directly the values of $g_{1}(r)$ to magnetostriction and those of $g_{2}(r)$ to the magnetocrystalline energy constant $K$ for cubic materials. It has also been used to predict the existence in ferromagnetic materials of a new kind of anisotropy called surface anisotropy, whose order of magnitude is $1 \mathrm{erg} / \mathrm{cm}^{2}$ and which depends on the angle of the spontaneous magnetization with the surface.

In case (b) one associates with each interstitial atom an energy $W \cos ^{2} \varphi$, where $\varphi$ is the angle between the link and the local direction of the spontaneous magnetization.

Lastly, in case (c) on associates with each atom on a $B$ site an energy $W \cos ^{2} \varphi$, where $W$ depends on the nature of the atom and $\varphi$ is the angle between the local direction of spontaneous magnetization and the ternary axis defining the symmetry of the six atoms surrounding the $B$ site.

The dependence of the intensity of the observed effects on the atomic concentration varies from one case to another. Assume for example a very small concentration $C_{A}$ of $A$ atoms or of interstitials. The effects are proportional to $C_{A}^{2}$ in case (a) and to $C_{A}$ in cases (b) or (c).

\section{MAGNETIC AND MECHANICAL DIRECTIONAL ORDERS}

When an ideal solution, made of $C_{a} A$ atoms and $C_{b}$ $B$ atoms $\left(C_{a}+C_{b}=1\right)$ undergoes at temperature $T_{1}$ the effect of either a high magnetic field or a simple tension $P$, a sudden-quenching to temperature $T_{0}$ creates a uniaxial anisotropy described by an energy density $C, \cos ^{2} \varphi$; where $\varphi$ represents the angle of the spon- taneous magnetization and the direction of the magnetic field or of the tension. According to the case, one has

$$
C_{s}=\frac{n C_{a}{ }^{2} C_{b}^{2} L_{0} L_{1}}{15 V R T_{1}} \text { or } C_{s}=\frac{3 C_{a}^{2} C_{b}^{2} L_{0} D_{0} P}{5},
$$

where $R=$ gas constant; $n=$ number of nearest neighbors; $V=$ atomic volume; $g$ and $X_{0}$ shear and compressibility moduli; $D_{0} / r_{0}$ is 4 times the deviation from Vegard's law expressed in relative units. $L_{0}$ and $L_{1}$ are the values at temperatures $T_{0}, T_{1}$, respectively, of a function defined by

$$
L=N\left(l_{a a}+l_{b b}-2 l_{a b}\right),
$$

where $N$ is Avogadro's number and $l_{a a}, l_{b b}, l_{b a}$ the values of $l$ for the various links.

Although the validity of the theory of directional order is no longer questioned, there are very few pertinent numerical data to illustrate it. The available data deal mostly with the product $L_{0} L_{1}$, and the values found are consistent with the values derived from magnetostriction through an analogous theory. In particular very little is known on the temperature dependence of $L$. With the hypothesis of a temperature dependence similar to that of the magnetostriction constants one may derive from the study of ferronickels a value of $L_{0}$, in agreement with that deduced in one hand from the concentration dependence of magnetostriction and on the other hand from the mechanical directional order which simply gives $L_{0}$.

\section{DIRECTIONAL ORDER AND ORDINARY SUPERLATTICES}

The link energies $w(l)$ defined in the foregoing are generally very small (of the order of $10^{-15} \mathrm{erg}$ ) compared with $k T\left(1.4 \times 10^{-13} \mathrm{erg}\right.$ at $\left.1000^{\circ} \mathrm{K}\right)$ so that the change of a link probability with its orientation is very small of the order of a few percent.

If this dependence on orientation were 20 or 30 times larger as it happens in some mixed cobalt ferrites, the short-range directional order would be replaced by a long-range order, of a cooperative character, i.e., a true uniaxial orientation superlattice (rhombohedral or tetragonal), remaining stable up to some transition temperature, at which the ordering vanishes.

In principle one should always be able to transform a directional ordering into an orientation superlattice by lowering sufficiently the temperature of the heat treatment. In practice, this is not possible since the lowest temperature of the heat treatments must be such as to allow mobility of the atoms in the lattice (about $400^{\circ} \mathrm{C}$ for ferronickels).

There is however an exception, that of magnetite (spinel structure) whose $B$ sites are equally occupied by $\mathrm{Fe}^{+++}$and $\mathrm{Fe}^{++}$ions, respectively. The electrons generating the reaction

$$
\mathrm{Fe}^{++}+e^{-} \rightleftharpoons \mathrm{Fe}^{++}
$$


are still active at $100^{\circ} \mathrm{K}$. The directional ordering can thus be transformed into a uniaxial orientation superlattice, whose axis may be chosen at will, by a suitable orientation of the magnetic field applied during the heat treatment.

There is of course a competition between directional ordering and ordinary superlattices when the latter appear. Thus in a 50-50 ferrocobalt, a superstructure appears at $720^{\circ} \mathrm{C}$ and affects a relative volume, which increases as the temperature is lowered. The disordered volume fraction, in which directional ordering may develop, decreases with the temperature: this explains Ferguson's results ${ }^{5}$ showing that constant $C_{s}$ decreases from 6 to $2.5 \mathrm{ergs} / \mathrm{cc}$, when the temperature decreases from 575 to $425^{\circ} \mathrm{C}$, despite the fact that the expression of $C_{s}$ indicates an increase of $C_{s}$ as the factor $\left(L_{1} / T_{1}\right)$ increases.

\section{MECHANICAL HEAT TREATMENT}

Although directional ordering due to this treatment has been predicted in the early stages of the theory, ${ }^{6}$ its experimental evidence due to Vergne ${ }^{3}$ is much more recent. The difficulty here is the necessity to avoid creep. Vergne has submitted a 55-45 ferronickel wire, at $500^{\circ} \mathrm{C}$, to a tension varying from 0 to $2.5 \mathrm{~kg} / \mathrm{mm}^{2}$, and measured the area between the magnetization curves before and after treatments. Assuming that the easy axes of magnetization are isotropically distributed at zero field, this area corresponds to half the anisotropy energy $C_{s}$ of a disk under the same tension. One thus finds $C_{s}=1400 \mathrm{erg} / \mathrm{cc}$.

\section{MAGNETIC AFTEREFFECT}

Let us now examine the effects of atomic diffusion on the magnetic properties. These effects are confined to a temperature range about $T_{a}$. Indeed, below this range diffusion is too slow to produce any perceptible effects. Above it, diffusion is so fast that thermodynamic equilibrium is always obtained.

Let us, for example, consider the case of $\mathrm{Fe}-\mathrm{C}$ at temperature ranges about -20 or $+30^{\circ} \mathrm{C}$, and let us assume that initially the interstitial carbon atoms are randomly distributed over the three kinds of available sites. If the spontaneous magnetization is kept in a fixed direction, the carbon atoms tend to concentrate

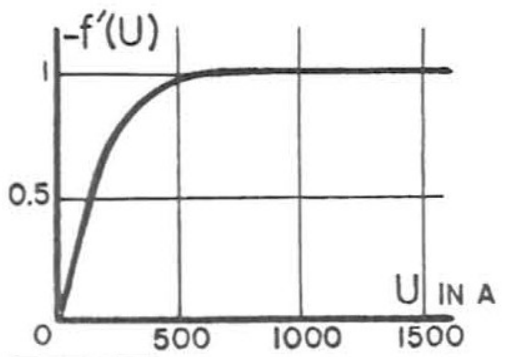

Fig. 1. The dependence of the restoring force due to the stabilization for a $90^{\circ}$ wall, $f(u)$, on the displacement of the wall, $U$.

${ }^{5}$ E. T. Ferguson, Comm. Colloque Int. Magnetisme, Grenoble, 1958.

${ }^{6}$ Louis Néel, J. phys. radium, 15, 225 (1954).

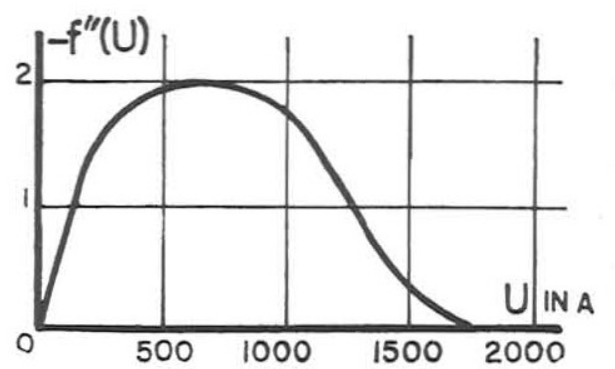

FIG. 2. The dependence of the restoring force due to the stabilization for a $180^{\circ}$ wall, $f^{\prime \prime}(u)$, on the displacement of the wall $U$.

on those sites for which the energy $W^{\prime} \cos ^{2} \varphi$ is minimum If the spontaneous magnetization stays along direction $\left(\alpha^{\prime}, \beta^{\prime}, \gamma^{\prime}\right)$ during a time sufficiently large in comparison with the relaxation time $\theta$, one can show the energy of uniaxial anisotropy given by

$$
E_{d}=-W_{0}\left(\alpha^{2} \alpha^{\prime 2}+\beta^{2} \beta^{\prime 2}+\gamma^{2} \gamma^{\prime 2}\right),
$$

is obtained with reference to the instantaneous direction $(\alpha, \beta, \gamma)$ of the spontaneous magnetization. Constant $W_{0}$ is connected to $W$ and to the atomic concentration $C$ of carbon by the relation

$$
W_{0}=C W^{2} / 3 k T \text {. }
$$

It is possible by quenching below $T_{a}$ to preserve the directional ordering of carbon atoms and to measure the corresponding anisotropy. Rathenau has thus found $W=0.79 \times 10^{-15} \mathrm{erg}$. This energy $W_{0}$ tends to maintain the spontaneous magnetization in the directions where it has settled for a long time.

These methods apply to variations in the magnetization, regardless of their origin, whether it is rotation or wall motion. It is wall motion, however, that has attracted the greatest interest among theoreticians and experimentalists alike.

Assume, for example, that after a Bloch wall has occupied a given position for a long time, we manage to displace it very suddenly by amount $U$. Due to the stabilization processes, the wall undergoes a restoring pressure, designated by $W_{0} f^{\prime}(U)$ or $W_{0} f^{\prime \prime}(U)$, for a $90^{\circ}$ or $180^{\circ}$ wall, respectively. The theory shows that in the case of iron these two curves are as given by Figs. 1 and 2.*

The aftereffect pressure tends, for large displacements, towards the limiting value $-W_{0}$ for $90^{\circ}$ walls and to zero for $180^{\circ}$ walls. This latter value can be easily explained, since in the largest part of the domain swept by the wall displacement, the magnetization simply changes its direction, and thus the energy $E_{d}$ of local stabilization remains unchanged.

In general, when an initial state $(t=0)$ characterized by an isotropic distribution of carbon atoms (as can be

* All figures in this paper are reproduced from the article of P. Brissonneau [J. Phys. Chem. Solids 7, 22 (1958)] by permission of Pergamon Press, Inc. 
obtained in principle by suitable demagnetization) is followed by a displacement $u(t)$, the theory shows that the pressure $P(t)$ exerted on the wall is given by

$$
P(u, t)=-W_{0} \int_{0}^{t} f(U) g(t-\tau) d \tau,
$$

where $U(t)=u(\tau)-u(t), f$ is equal to $f^{\prime}$ or $f^{\prime \prime}$ according to the nature of the wall, and where

$$
g(t-\tau)=\int_{0}^{\infty} p(\theta) \exp \left(-\frac{t-\tau}{\theta}\right) d \theta .
$$

Here $p(\theta) d(\theta)$ is the probability for the time constant to lie in the interval $(\theta, \theta+d \theta)$.

The position $u(t)$ of a wall, separating two domains of respective magnetizations $J_{1}$ and $J_{2}$, in a magnetic field $H(t)$ and submitted to a restoring pressure $R(u)$, satisfies the integral equation

$$
\mathbf{H} \cdot\left(\mathbf{J}_{1}-\mathbf{J}_{2}\right)+R(u)+P(u, t)=0 .
$$

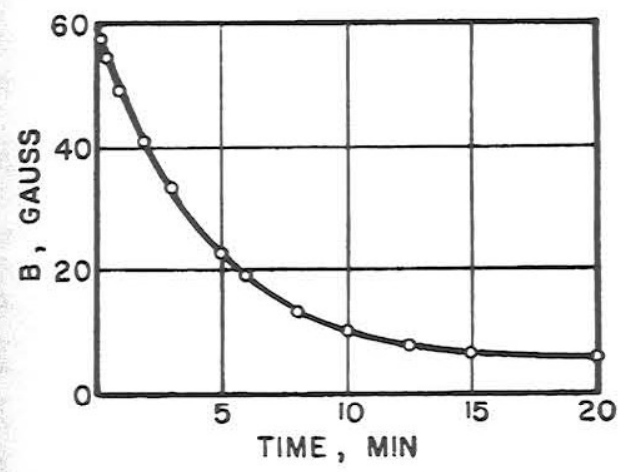

FiG. 3. Disaccomodation of the induction of an $\alpha$-iron at $-27.3^{\circ} \mathrm{C}$, $H=32 \mathrm{~m} \phi \mathrm{e}, C=4.6 \times 10^{-3} \%$.

The various conclusions of the theory we have just outlined are confirmed by experiment. Let us mention in particular the very careful and very accurate measurements of Brissonneau.

The first results are related to the so-called disaccommodation of the susceptibility or of the magnetic induction: following demagnetization, the sample is kept in zero field during a period $t$, at the end of which a field $H$ is suddenly switched on, and the instantaneous variation $B(H, t)$ of the induction [proportional to $u(t)$ ] is measured. The theory is very simple, the variables are separable and the results are

where

$$
\begin{aligned}
& P(u, t)=W_{0} f(u) G(t), \\
& G(t)=\int_{0}^{t} g(t-\tau) d \tau .
\end{aligned}
$$

Figures 3 and 4 indicate the time dependence of $B$ for $H=32 \mathrm{~m} \phi$ at $-27.3^{\circ} \mathrm{C}$ and with $C=46.10^{-6}$. A simple inspection of these curves suggests the diffi-

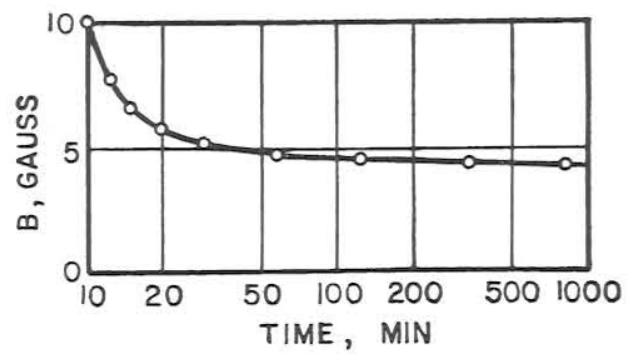

FIG. 4. Disaccomodation of the induction for very long times of an $\alpha$-iron at $-27.3^{\circ} \mathrm{C}, H=32 \mathrm{~m} \phi \mathrm{e}, C=4.6 \times 10^{-3} \%$.

culties of interpretation if one assumes a unique time constant. Figure 5 gives the curves $B(H)$, using $t$ as a parameter. Consider two of these curves, corresponding to $t=0.70 \mathrm{~min}$ and $t=1000 \mathrm{~min}$, for example. For the same value of $B$, one curve may be deduced from the other by a translation of an amount $H(t)$, which is the aftereffect field related to $f(u)$, for an isolated wall, by the relation

$$
H(t)=\frac{W_{0}}{J_{s}} f(u) \cdot G(t) .
$$

Experiments show (Fig. 6) that $H(t)$, proportional to $B$ for small values of $B$, reaches a maximum value of the order of $80 \mathrm{~m} \phi \mathrm{e}$ for $B \sim 50$ gauss, and decreases

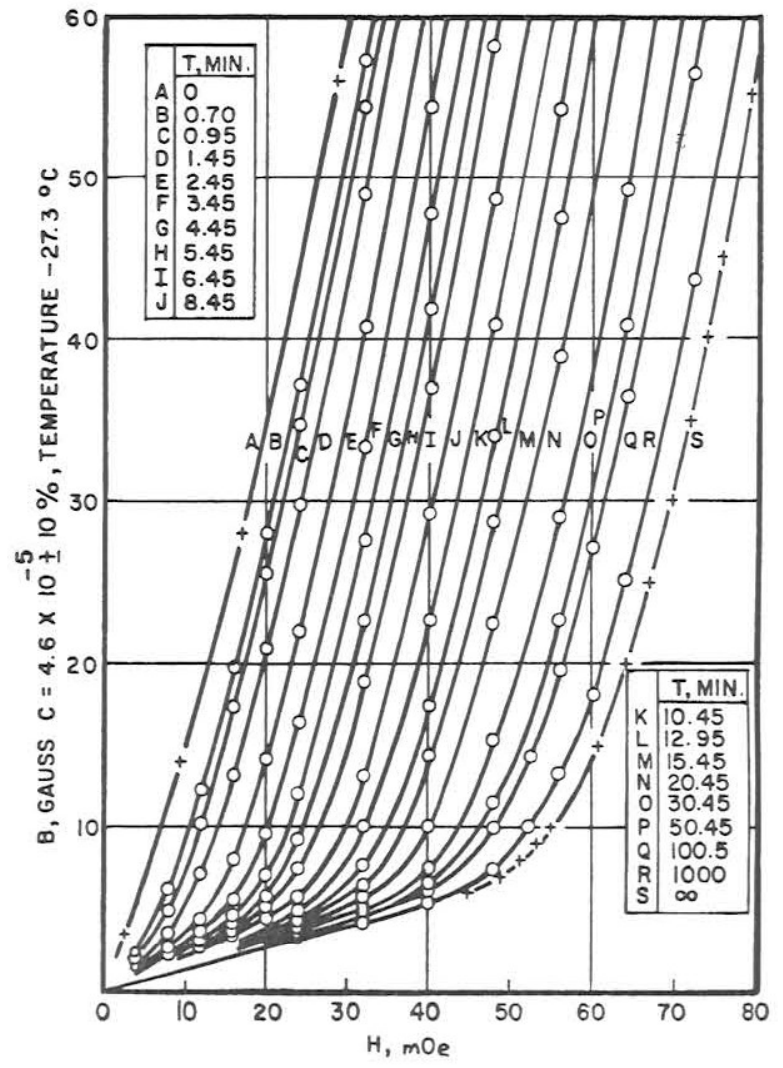

FIG. 5. Isochronic magnetization curves $B(H, t)$ or an $\alpha$-iron, where the induction $B$ is plotted as a function of $H$, taking the time $t$ as a parameter. 
towards a constant value of $30 \mathrm{~m}$ фe for large inductions (2000 to 6000 gauss). The shape of this curve shows, as could be expected, that the sample contains both $90^{\circ}$ and $180^{\circ}$ walls. The maximum value is typical of the $180^{\circ}$ walls while the $90^{\circ}$ walls are responsible for the nonzero limiting value.

From the experimental values of the maximum aftereffect field $\left(W_{0} / J_{s}\right)$ and from the carbon concentration $C$, Brissonneau has deduced the value $W=0.71$ $\times 10^{-15}$ ergs very near of the value $0.79 \cdot 10^{-15}$ ergs derived from a direct measurement of the anisotropy due to directional ordering. This remarkable agreement illustrates the close relationship between magnetic aftereffect on one hand and on the other hand directional ordering and orientation superlattices.

Brissonneau's experiments correspond to a complex mixture of $90^{\circ}$ and $180^{\circ}$ walls of diverse orientations. They do not prove definitely that the aftereffect pressure vanishes for large displacements of $180^{\circ}$ walls. A more conclusive evidence has recently been provided by Rathenau and his co-workers who analyzed an $\mathrm{Fe}-\mathrm{Si}$ monocrystal $\left(3 \%\right.$ Si) containing only $180^{\circ}$ walls. After reaching a sharp maximum the aftereffect pressure decreases to zero for values of the induction ranging from 50 to 200 gauss. These experiments have also

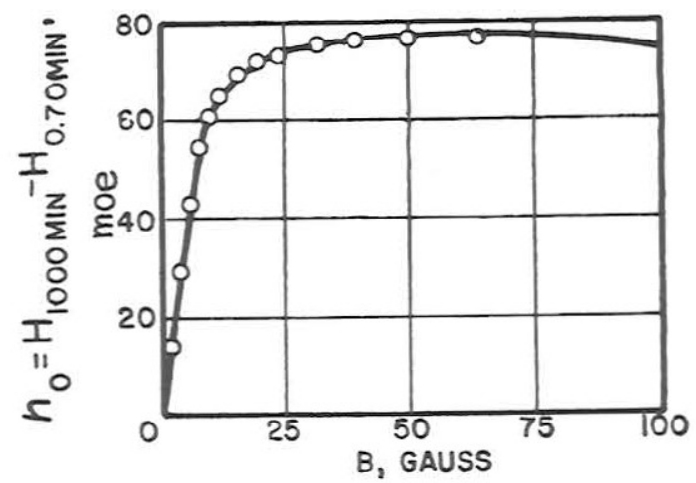

(a)

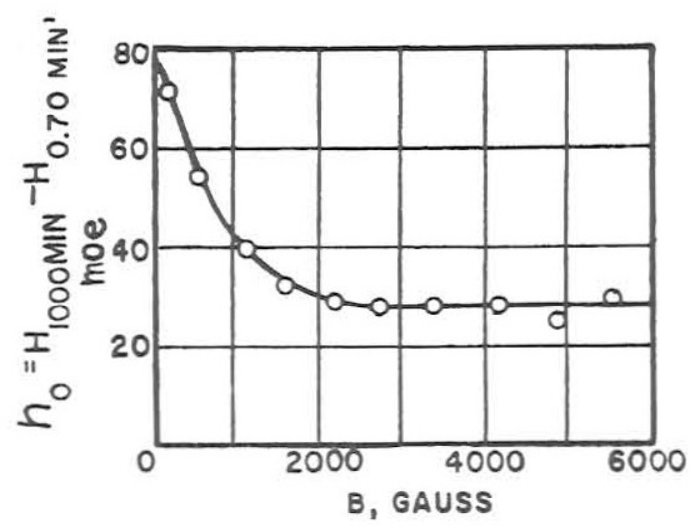

(b)

FIG. 6. Variation of the aftereffect field of an $\alpha$-iron as a function of $B$, at $-33.5^{\circ} \mathrm{C}, C=7.5 \times 10^{-30} \%$, (a) for small values of $B$, (b) for large values of $B$.

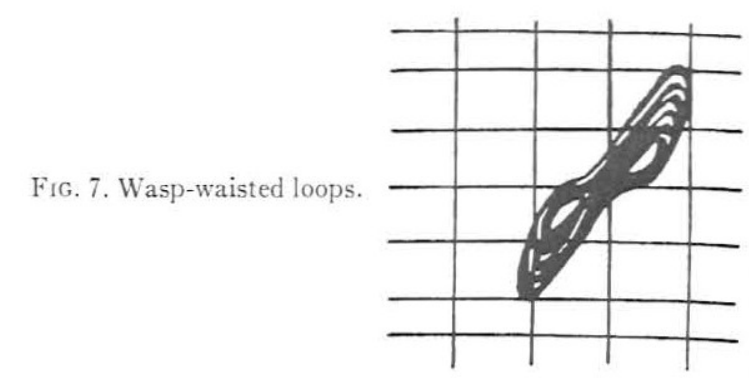

yielded Bloch wall widths departing by less than $10 \%$ from the values calculated 15 years ago by Néel.

\section{WASP-WAISTED HYSTERESIS LOOPS}

Theory shows and experiment confirms that after stabilization, the aftereffect field becomes very nearly constant ( $80 \mathrm{~m} \phi \mathrm{e}$ in the preceding example) for induction values ranging from 25 to 100 gauss. This aftereffect field is always directed against the applied field. When one applies an alternating field to describe hysteresis cycles between $+H_{m}$ and $-H_{m}$, the situation can be described by the superposition of an aftereffect field $\mp H_{f}$ to the applied field $\pm H$ ( $H$ positive). The positive half-loop is shifted by an amount $+H_{f}$, and the negative one by an amount $-H_{f}$. Thus is obtained a wasp-waisted loop, in the shape of bowtie, as illustrated in Fig. 7 . As the time interval $t_{2}-t_{1}$, between the instant $t_{2}$ of observation and the instant $t_{1}$ at which the ac field is swicthed on, increases the stabilization effects disappear progressively and the cycles resume their classical shape.

The analogy is remarkable between these waspwaisted loops and the hysteresis cycles of some alloys (perminvar and permalloy types) and of ferrites containing cobalt oxide. We are here in presence of the same fundamental phenomenon, and more specifically of variations (a) and (c) mentioned at the beginning of the paper. In this case the stabilization takes place only at relatively high temperatures, higher than $400^{\circ} \mathrm{C}$. But once the stabilization has been obtained, and followed by quenching, its effects may be maintained indefinitely at room temperature. The waspwaisted cycles become a permanent property of the material, while they last only 10 to $30 \mathrm{~min}$ for $\mathrm{Fe}-\mathrm{C}$ at $-21^{\circ} \mathrm{C}$.

\section{CONCLUSIONS}

Thus is established a solid experimental basis for the theory of diffusion aftereffect, as well as for the analogy between this theory and that of magnetic and mechanical orientation phenomena, due to directional ordering. These theories show also that very simple assumptions and methods may lead to interesting conclusions, in agreement with experiment. 